\title{
Digital Foam Interaction Techniques for 3D Modeling
}

\author{
Ross T. Smith * \\ Bruce H. Thomas ${ }^{\dagger}$ \\ Wayne Piekarski ${ }^{\ddagger}$ \\ University of South Australia
}

\begin{abstract}
Digital Foam is a new input sensor developed to support clay like sculpting and modeling operations. We present techniques facilitating navigation and manipulation operations performed using Spherical Digital Foam as a sole input device. Our free-form sculpting technique allows manipulation of new and existing 3D models using accumulated sculpting like motions. Digital Foam's multi-point pressure sensitive surface captures the separate locations of a user's fingertips allowing controlled manipulation of multiple model vertices simultaneously. Additionally, we developed a technique that allows the camera view and zoom to be controlled by applying varying pressure to the Digital Foam surface. Furthermore, we have designed a menu system tailored for operation using Spherical Digital Foam as a sole input device using both the internal orientation sensor and the pressure sensitive surface.
\end{abstract}

A new higher resolution Spherical Digital Foam input device with 162 unique pressure sensors is presented. This is a significant improvement in comparison to the previous Spherical Digital Foam version with only 21 sensors. We discuss the design issues and how an increased resolution affects the operation and design of the algorithms used. We propose a new dynamic button allocation technique made possible using the new high resolution Spherical Digital Foam. Finally, we performed a trial study using the new 162 sensor Spherical Digital Foam input device to evaluate elements of the menu system.

CR Categories: D.2.2 [Software Engineering]: Design Tools and Techniques-User interfaces; H.5.2 [Information Interfaces and Presentation]: User Interfaces-Prototyping I.3.6 [Computer Graphics]: Methodology and Techniques-Interaction techniques

Keywords: Digital Foam, 3D Input Device, Interactive Modeling, Augmented Reality, Virtual Reality, Interaction Techniques

\section{Introduction}

Creating and capturing 3D models is performed by graphic artists, industrial designers, researchers and those of many other disciplines. Current 3D modeling applications, such as Autodesk's 3ds Max or Maya, perform surface modeling using a range of input devices including keyboard, mouse and tablets. Manipulation techniques are based around mathematical operations to alter surface shape and require extensive training to master. Our goal is to develop a range of techniques that support intuitive $3 \mathrm{D}$ modeling in-

*e-mail: ross@r-smith.net

†e-mail: thomas@cs.unisa.edu.au

‡e-mail: wayne@tinmith.net

Permission to make digital or hard copies of all or part of this work for personal or classroom use is granted without fee provided that copies are not made or distributed for profit or commercial advantage, and that copies bear this notice and the full citation on the first page. To copy otherwise, to republish, to post on servers or to redistribute to lists, requires prior specific permission and/or a fee. VRST'08, October 27-29, 2008, Bordeaux, France

Copyright 2008 ACM ISBN 978-1-59593-951-7/08/10 ...\$5.00 teractions based on free-form sculpting operations like those used when working with modeling clay. By using the clay sculpting metaphor for the interaction technique design, we endeavored to leverage people's pre-existing understanding of physical clay modeling properties. Digital Foam allows a computer to capture squeezing operations similar to those used when working with clay.

Figure 1 depicts a user manipulating the Spherical Digital Foam input device and viewing the resultant 3D model on a monitor. Although we have been investigating the use of this input device in a fishtank VR mode, the Spherical Digital Foam is fully functional in an immersive VR mode. Spherical Digital Foam provides both command entry and direct manipulation interactions. It is also well suited to virtual cloaking using virtual 3D graphical objects to overlay the physical view of the input device.

Recently touch based input devices have become very popular within consumer electronics. The use of touch screens on mobile phones and $\mathrm{mp} 3$ players is extensive, and more recently the new Microsoft surface computing ${ }^{1}$ has emerged. We envisage Digital Foam offers a new dimension to touch based surfaces that can be applied to 3D modeling.

Previously presented was the technical construction of Digital Foam [Smith et al. 2008] a multi-touch, pressure sensitive surface. Two prototypes were presented, a flat surface with 100 sensors and a spherical input device with 21 sensors creating the Digital Foam surfaces. Also with the presentation of Digital Foam, a set of initial techniques and application ideas were proposed including sculpting, menu navigation and camera view control.

To reveal Digital Foam's potential, we begun by focusing our research efforts on developing, implementing and testing a number of interaction techniques to support 3D modeling. For the purposes of our investigations we have made the assumption that Digital Foam

\footnotetext{
${ }^{1}$ http://www.microsoft.com/surface/
}

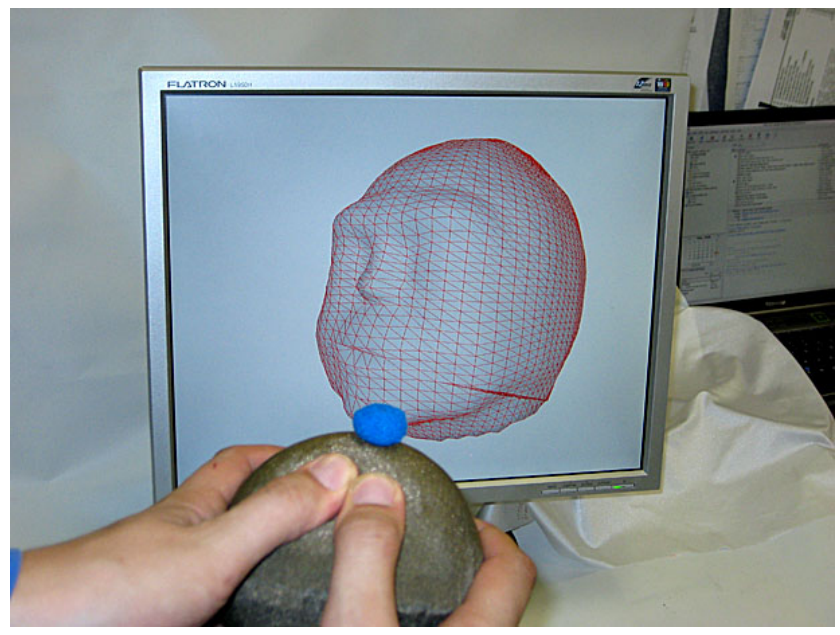

Figure 1: User sculpting 3D model using Digital Foam. 
will be used as a sole input device. This was chosen so as the modeling and interactions can be performed without the need for a keyboard or mouse, a common requirement when using virtual and augmented reality systems. This also removes the need for the user to put the input device down freeing their hands to use a keyboard or mouse. Although speech input is a possible command entry technology, we focused on a single device for command entry and direct manipulation of the object's surface.

We present six contributions to the field of human computer interaction. First, we extend the previously proposed sculpting technique to allow surface manipulations of existing 3D models (from simple 3D shapes like spheres to complex 3D models such as human heads) and allow accumulative operations to be performed through the use of clutching [Hinkley et al. 1998]. Secondly, we demonstrate the implementation of the unique orbital camera view control proposed in [Smith et al. 2008]. A user controls the current camera position by pressing one finger on the Digital Foam surface and can alter the cameras zoom based on the fingers pressure. Thirdly, a menu system is presented (navigation and menu item selection) based on the ideas proposed in [Smith et al. 2008]. We further extended this technique to use the Digital Foam sensor for click operations removing need for additional input devices such as a keyboard or mouse. Fourthly, we present the half hemisphere operating technique to allow the user to grasp the Spherical Digial Foam and perform free-form modeling and menu options more intuitively. Fifthly, we present a new high resolution Spherical Digital Foam input device and discuss the improvements and issues introduced as the resolution is increased. Benefits of this improved resolution allow the user to perform more complex sculpting operations and also inspired the conceptualization of a new dynamic command entry allocation technique not possible on the lower resolution Digital Foam version. Finally, we have performed an initial trial study to assess the qualitative aspects of the menu control system. This has measured aspects of the user experience and will facilitate future designs directions.

\section{Related Work}

Many input devices have been constructed specifically for controlling interactions in 3D environments. Even with the availability of these new input devices, numerous existing application use 2D input devices such as a mouse to manipulate $3 \mathrm{D}$ information. Human hands normally control 3 degrees of freedom (DOF) positioning and 3 DOF orientation, but to make interfaces more understandable for users, the number of axis are sometimes reduced [Deering 1995]. Virtual reality systems attempt to provide a natural mapping between the user's actions and human computer interface, such as Virtual Clay [McDonnell et al. 2001] an interactive free-form modeling environment. Virtual Clay [McDonnell et al. 2001] supports clay like manipulations and intuitive sculpting with physics based responses and haptic feedback using a Phantom device [Massie and Salisbury 1994].

A common feature of virtual environment input devices is the creation, manipulation, and navigation of 3D geometries. The "two 4 - six" input device [Kulik et al. 2006] is designed to support 3D manipulations with 6 DOF. Orientation is tracked with gyroscopes, and a rocker leaver with an elastic touchpad is employed to control rotation, translation and manipulation operations. A second custom device is the Cubic Mouse [Fröehlich and Plate 2000], a cubic shaped input device with three rods that protrude out of the faces of a cube. Users push and pull on the rods corresponding with the $\mathrm{X}, \mathrm{Y}$ and $\mathrm{Z}$ axis to interact with the system. The input device itself may be 6 DOF tracked to allow the rods to be registered with a virtual environment.
Current investigations into malleable surfaces [Milczynski et al. 2006; Vogt et al. 2004; Kamiyama et al. 2004] are similar conceptually to Digital Foam. A camera is mounted underneath a silicon membrane. The silicone membrane employs colored dots on its surface that are tracked by the camera. Deformations can then be determined for a reconstruction of the silicon's surface shape. A limitation of these malleable surfaces is that to construct a spherical prop where all surfaces can be deformed is mechanically difficult and perhaps not possible.

Buxton presents a taxonomy of input devices [Buxton 1995] describing differences between mechanical and touch based input devices. Hinkley et al. presented Touch-Sensing Input Devices [Hinkley and Sinclair 1999], where the use of a trackball and mouse with additional touch sensors was described. Hinkley et al. also developed the On Demand Interface technique which uses touch information to fade parts of the screen in and out depending on user actions, a further classification of tactile input technologies is also provided.

Many 3D surface manipulation techniques have been developed to support artistic and intuitive user interface techniques. Pinch gloves [Piekarski and Smith 2006] capture finger presses and pose. Cyber Gloves $^{2}$ use 18 or 22 flexible sensors to track a user's fingers and hand pose. Used in conjunction with the Cyber Grasp additional haptic feedback is applied to the fingers using actuators. One disadvantage is the gloves are bulky and require setup time while attached to the users body. Schkolne et al. [Schkolne et al. 2001] used Cygber Gloves and the Responsive Workbench [Kruger and Fröehlich 1994] to create a free-form 3D modeling system. Their system "Surface Drawing" tracks the user's hand locations and Cyber Gloves to allow stroke based drawing to be performed. This system is of particular interest as it is designed to support creative expression in a three dimensional space. Their techniques allow sketching by tracking a user's arm location and hand pose. The authors compare creating a stroke in 3D space to drawing a line on a piece of paper. Unlike Surface Drawing, Digital Foam does not require $6 \mathrm{DOF}$ tracking as no path is required for the geometry creation. A fundamental difference between Surface Drawing and Spherical Digital Foam techniques is when using Digital Foam, the user starts with a solid geometry such as a sphere or existing model and performs surface manipulations to create the final shape rather than the stroke based approach.

Jung et al. completed a field study observing artistic modeling techniques including Computer Aided Design, clay modeling, wood carving and glass crafting [Jung et al. 2004; Jung et al. 2005]. They observed these techniques to conceptualize and develop Spray Modeling. Spray Modeling uses a tracked physical spray gun as a tangible prop. Models are created in three steps, an initial 3D frame drawing, volume spraying, and air spraying for smoothing. Hinkley et al. employed physical props to represent a 3D skull and cutting plane model [Hinkley et al. 1994]. User actions were tracked using a Polhemus ${ }^{3}$ tracking system and mapped allowing a user to explore the inner geometry of the skull model. Digital Foam encompasses many of the design philosophies presented by Schkolne, Jung and Hinkley. Digital Foam is based on a tangible prop the user holds and manipulates. Initially we have chosen a spherical prop as a generic shape and perform a logical mapping between the spherical shape and the virtual model. There is no technical reason more complex shapes could not be manufactured with a Digital Foam covering .

Numerous different menu systems have been used in Virtual and Augmented Reality systems. Blaskö et al. developed a pull down menu interface for wearable computing [Blaskö and Feiner 2002].

\footnotetext{
${ }^{2}$ http://www.immersion.com/

${ }^{3}$ http://www.polhemus.com/
} 
A user can select hierarchical menu options by running their finger along either the horizontal lower edge or predefined vertical strips of the touchpad. Bowman et al. used pinch gloves to control their TULIP menu system [Bowman and Wingrave 2001]. Menu items are mapped to each finger in the virtual environment. Circular menus have been employed in virtual environments. HoloSketch [Deering 1995] uses a 3D pie menu with concentric menu items that can be activated with a wand. Liang et al. presented the JDCAD 3D modeling system [Liang and Green 1993] that uses a spherical and ring menu for object selection. The idea is further evaluated and developed by Gerber and Bechmann [Gerber and Bechmann 2004; Gerber and Bechmann 2005] into a hierarchical spin menu as a context menu in a VR environment.

Reitmayr et al. presented the iOrb [Reitmayr et al. 2005], a handheld input device that tracks orientation. Menu operations are controlled by first mapping three orientation values to a $2 \mathrm{D}$ coordinate system with orthogonal axis based on the user's current arm pose. These values are then mapped to different menu widget styles. Two selection methods were used, one uses a time-out value and the second uses a predefined threshold angle allowing menu selection operations.

\section{Free-form Sculpting and Clutching}

The goal of our Spherical Digital Foam input device is to develop interaction techniques that support 3D modeling operations. Since Digital Foam's initial publication of [Smith et al. 2008], we have implemented the proposed free-form interaction technique and discovered additional procedures that increase the functionality of free-form modeling. Firstly we have added the option to load existing 3D models into the application. A user can now begin the modeling process with either the reconstructed shape generated by the hardware device or by loading an existing 3D model. To allow manipulations to be performed on existing models we map the Digital Foam sensors locations to the model allowing semi-direct manipulation to be performed. We refer to the mapping as semi-direct because the input device shape is not the same as the 3D model. However a spatial mapping is maintained between the two.

To achieve the mapping between the 3D model and Digital Foam, a set of rays aligned with each of the conductive foam sensors are cast from the center of the $3 \mathrm{D}$ model to find the intersection points on the outer surface the 3D model (Figure 2(b)). Once each intersection point is found, an index to each vertex is stored for later use. The length of each Digital Foam sensor is mapped directly to these intersection points (as described in Equation 1) allowing the user to modify the 3D model by pressing on the Digital Foam surface. The new vertex location $\left(P^{\prime}\right)$ is found by translating the original position $P$ in the direction of the ray using the foam length as the scalar value.

$$
P^{\prime}=P *(s u * f l)
$$

$P=$ Intersection point on model's outer surface.

$f l=$ Current length of the foam sensor.

$s u=$ Normalized Digital Foam vertex location.

To increase the working area between sensor points we have applied an algorithm to find the closest surrounding vertices within a user defined radius. We calculate the surrounding vertices for each intersection point and store them in ascending order based on length from the intersection point $(P)$ to each vertex $(V)$ on the model. For each vertex $(V)$ within the user defined radius we find $V^{\prime}$ (the set of new vertex locations) by scaling the foam length $(f l)$ by length between the intersection point $(P)$ and the vertex $V$, see
Equation 2. The furthest vertex within the predefined radius has no modification, generating a curved indentation shape used to perform sculpting (Figure 2(d)).

$$
V^{\prime}=V *\left(\left(s u *\left(f l *|f d-v d|^{2} * c\right)\right.\right.
$$

$V=$ Current vertex.

$s u=$ Direction pointing out from the center of the model.

$f d=$ Length from the furthest vertex to $\mathrm{P}$.

$v d=$ Length from the current vertex to $\mathrm{P}$.

$c=$ Scale factor.

The Spherical Digital Foam employs a tilt based clutching mechanism to allow accumulative modeling operations. A user performs a sculpting operation by pressing the foam to the desired location, tilts the prop 20 degrees and releases their finger. Each of the vertex positions are stored and the process can be repeated (Clutching operation show in Figure 2(c)).

Manipulation direction (push in or push out the 3D virtual model) can be set allowing the inverse operation to be performed. We based this technique on the observation that artists commonly attach and detach clay to a physical model during its creation. The user can change the direction by toggling a menu option. The combined techniques discussed here allow the modification of vertices to be either additive or subtractive (Figure 2(d) shows a resulting sculpting operation operation depressing the left cheek).

Some general observations can be made about the design aspects of Digital Foam. Unlike Surface drawing [Schkolne et al. 2001] we start with a fixed volume and perform sculpting operations similar to [Piekarski and Thomas 2004]. We chose this design to emulate clay sculpting using a single solid piece of modeling clay. However, in the future full $6 \mathrm{DOF}$ tracking could be added to allow path capture.

\section{Half Hemisphere Operation}

When holding a Spherical Digital Foam input device, a user's fingers and thumb may cause depressions in more than one location on the foam surface. This is problematic when free-form modeling, as these could be interpreted as unwanted modeling gestures. For example, when a user performs sculpting operations at the front of the sphere using their thumbs, their fingers are located at the back of the sphere causing depressions at both the front and the back as shown in Figure 3(a).

We have developed a technique that divides the sphere's operation surface into two hemispheres, front and back. All vertices located on the front hemisphere relative to the user's view point remain active, while those behind are made inactive (Figure 3(b)). On initialization, the user specifies the front orientation and can not move their head position or orientation during operation ( Additional trackers are required on the users head and Digital Foam to achieve this). As the user rotates the Spherical Digital Foam input device the virtual model's orientation is updated in real-time using the internal orientation sensor. To maintain the half hemisphere operation, all vertices that are in front of the center point are flagged as active while those behind are inactive. This operation overcomes a significant user interface problem when operating Digital Foam; thus allowing easier operation and increased control during modeling. The half hemisphere operation can be applied to work in conjunction with other techniques allowing stacked operations to be performed. For example, half hemisphere operation can be used with sculpting or menu click operations. 


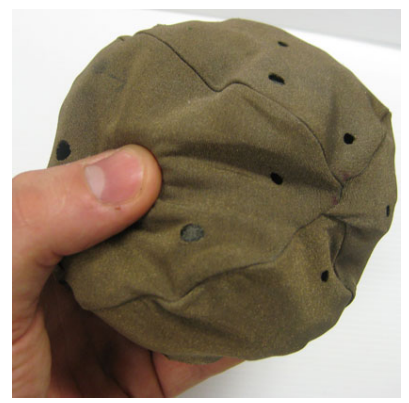

(a)

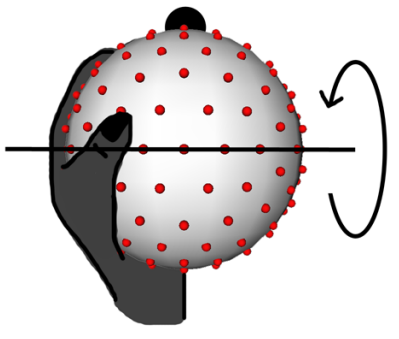

(c)

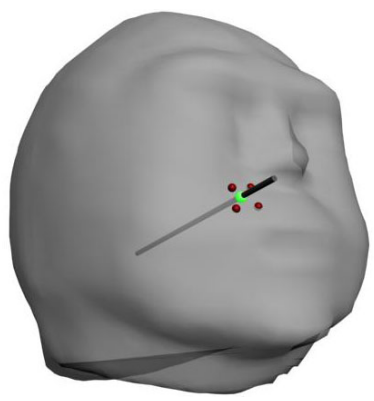

(b)

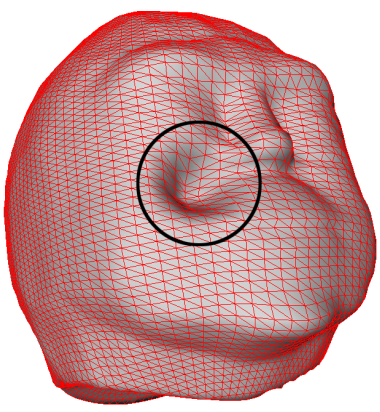

(d)
Figure 2: Free-form Sculpting Operations. (a) User sculpting Digital Foam. (b) Ray intersection and surrounding vertices calculation performed. (c) clutching tilt operation resets vertex locations. (d) Resulting geometry after single clutched free-form sculpting operation.

\section{Camera View Control}

A new camera view control technique has been developed allowing a user to quickly and intuitively move the virtual cameras position. Figure 4 depicts the operation of the Digital Foam sphere in the camera view control mode. While in the camera view control mode, a user touches any part of the surface of the sphere and the camera viewpoint will be shifted to the matching location. When multiple sensor readings (depressions) are detected, the foam sensor with the shortest value is used to determine the camera position. The direction of the camera is determined in a similar fashion to the orbital view algorithm [Koller et al. 1996]. A bounding sphere is created around the virtual model and the direction of the camera is set to look at the center of the object. The user can also control the zoom of the camera based on the pressure of the touch. As the user pushes on the Digital Foam harder the camera zooms in closer and as they release the zoom location returns.

\section{Menu Control}

A custom menu system was developed as the primary command entry technique used when operating the Spherical Digital Foam input device. The navigation of the menus is designed to be intuitive, quick and easy to use so as minimal user training is required. There are a number of challenges that need to be addressed to use Digital Foam as a sole input device for both command entry and direct manipulation. We refer to free-form sculpting, camera view and all modes of direct model interaction as interaction modes. We have implemented a technique that allows the user to transition from any interaction mode into a menu mode without using additional input devices.

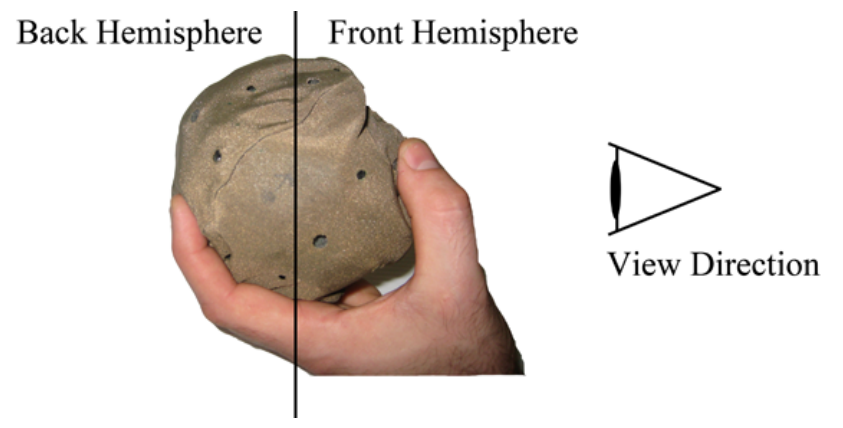

(a)
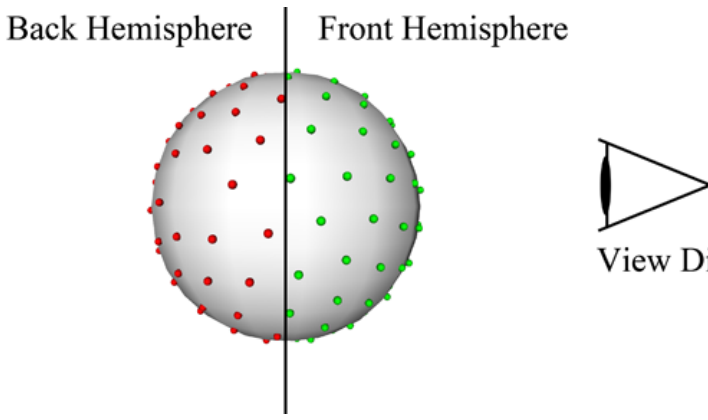

View Direction

(b)

Figure 3: Half hemisphere technique. (a) User performing sculpting with thumb, unwanted finger presses at back of sphere. (b) Half hemisphere correction being performed, active vertices shown on right side, inactive vertices shown on left side.

To transition from interaction mode to menu mode the user rotates the input device up-side-down so the roll or pitch is beyond a predefined threshold value (currently set to 90 degrees and shown in Figure 5(a)). Once in the menu mode, the user can navigate through menus by rotating the input device around the heading (vertical) axis.We currently have configured 10 menu options allowing the user to select different interaction modes, but as the number increases, we will add additional hierarchical menus. To scroll through menu options we have set the transition at 20 degree intervals. When the user rotates around the heading axis the selected menu option changes from one menu option to the next every 20 degrees (Figure 5(e)). Currently ten menu items are displayed on two rows with five menu items on each row. A transition from row one to row two occurs when the last item in row one is reached. By rotating the input device beyond the last item in row two a transition to the first row occurs.

Once the correct option is selected, a menu selection operation is required. To achieve this we use the Digital Foam sensors, by squeezing the input device with one or two hands a menu selection operation is performed. In software this is determined when the average value over all sensors drops below a predefined threshold and a click event is generated. Finally once the option has been selected and clicked, the menu is hidden and the selected interaction mode becomes immediately active.

To re-enter the menu mode, the input device orientation must first return so as rotation values are above the predefined threshold. Once this has occurred the device can be turned up-side-down again to enter menu mode. Figure 5 shows the different states of the menu selection operation.

One limitation of this technique is that when operating in modes that map the orientation sensor directly to the model, the menu 


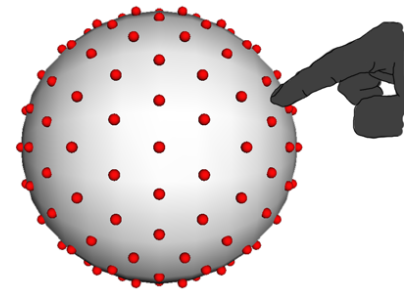

(a)

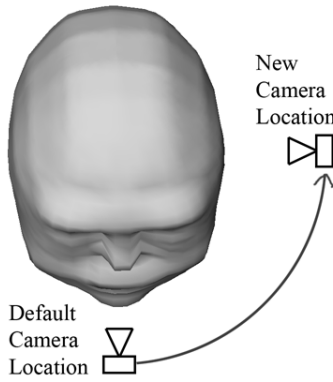

(b)
Figure 4: (a) User controlling the camera location with press location, and zoom with pressure. (b) Camera location transition.

mode may be accidentally entered. Although this is a limitation, we have found rotating around the heading is most commonly used for model navigation and both pitch and roll are unaffected until they pass the threshold value (currently set at 90 degrees).

\section{Common Techniques}

This section presents a collection of common interaction techniques, such as rotation and scale. The Spherical Digital Foam has the unique feature of pressure sensing of the user's interaction, and an explantation of how we exploit this feature is presented. Finally, a description of an orientation visual landmark is given, and the file handling features are explained.

\subsection{Rotation}

We use two model rotation control modes. The first uses a direct mapping between the values of internal orientation sensor and the 3D model. The updating model rotation can be used in conjunction with other techniques such as free-form sculpting to adjust the current view angle. A menu option can be toggled to turn rotation on and off, however this mode is stateless and the model can not be set to user defined position once this interaction mode is left.

To overcome this problem we added a second rotation control mode that allows a default rotation angle to be set. When using set rotation no rotation transformations are performed until the user begins squeezing the Digital Foam input device. When the desired operating angle is selected the user stops squeezing the input device and this angle is recorded and used as a default model orientation for all other interaction modes.

\subsection{Scale}

Scale functions of the model were easily added, we have eight separate scale operation. Each is activated by squeezing the Digital Foams surface to directly alter the scale value. The scale can be altered on $\mathrm{X}, \mathrm{Y}$ or $\mathrm{Z}$ axis separately or a combined operation where the overall model's size is altered. The direction of scale can also be toggled via the menu.

\subsection{Speed and Pressure}

Digital Foams unique pressure sensing surface can gather pressure data that may be process with different methods depending on the task being performed. Capturing the speed of a press for each separate sensor on the Digital Foam surface is possible. This is done by keeping a buffer for each foam sensor that records its distance and

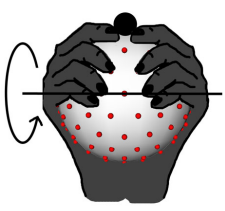

(a)

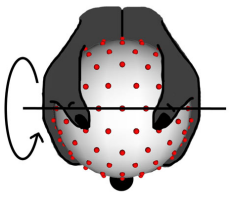

(c)

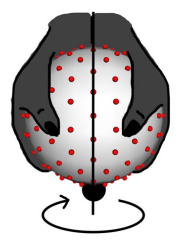

(e)

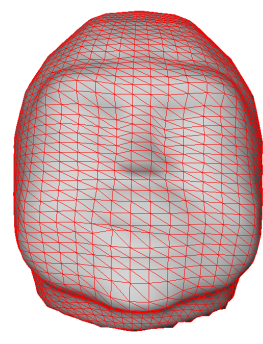

(b)
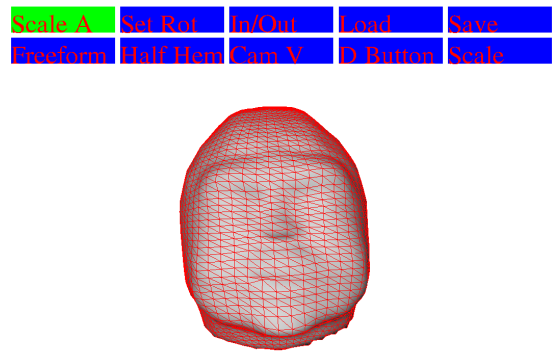

(d)

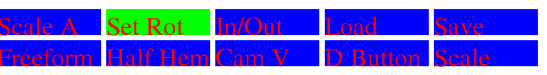

Figure 5: Menu operating procedure. (a) User Interaction pose. (b) Interaction mode (c) User rotates prop up-side-down. (d) Menu is displayed. (e) User rotates around heading to select different menu options. (f) Scrolling menu option.

a time value. For example, keeping a ring buffer with a size of 20 is adequate to capture a range of button press/release speeds. We currently calculate this data to confirm the feasibility of this technique, however we have not applyed it to specific modeling techniques.

One limitation of the physical properties of the conductive foam is intense compression of the foam sensors has a slow return when depressed beyond $80 \%$ of its original size [Brady et al. 2005]. Currently we avoid pressing the sensors too hard, this could be further prevented with a mechanical stop.

\subsection{Orientation Marker}

When using the menu system, free-form sculpting or the camera view technique, it is useful to have a marker on the physical device to identify the top of the sphere. We have attached a physical marker to the input device. The physical marker can be seen in Figure 1 immediatly above the user's thumbs on the Spherical Digital Foam input device. A matchnig software marker can also be toggled on and off via the menu. This simple technique provides a spatial reference between the physical prop and the 3D model. 


\subsection{Load and Save}

Existing models can be loaded into the scene as previously discussed. The entire scene graph can also easily be saved by selecting the appropriate menu option. Currently we save the file in SGIs Inventor format ${ }^{4}$ which is easily converted to many other model formats.

\section{High Resolution Digital Foam}

To support a 3D modeling system using Spherical Digital Foam we quickly recognized the density of the sensors relates directly to user control and the final resolution of the models that can be sculpted. The first prototype constructed [Smith et al. 2008] has 21 foam sensors, we found this adequate for testing and creating many of the new interaction techniques. However, we also planned to iteratively increase the resolution by changing the electromechanical design. There is an improvement in the hardware performance using design considerations we learn from a number of experimental prototypes.

Constructing a higher resolution prototype had two difficult technical boundaries to overcome. Firstly, constructing a foam surface in a sphere shape with a large number of sensors required custom construction techniques to be developed. The position of the sensors has to be placed evenly around the sphere's surface. To achieve this we used two well known techniques, a subdivision algorithm [Woo et al. 1998] and a repelling algorithm. The subdivision algorithm generates perfectly evenly spaced vertex location but only certain numbers of vertices are possible. The algorithm starts with one of the five platonic solids and is reduced by dividing each face into four new faces until the desired complexity is reached. By choosing different base platonic solids and performing different division levels there are a large number of evenly spaced vertex spacings that can be generated. Alternatively a repelling algorithm can generate almost evenly spaced points with $\mathrm{N}$ vertices. We used the repelling algorithm on the previously constructed 21 sensor prototype [Smith et al. 2008] and decided to use the subdivision technique on the 162 sensor prototype presented here. We chose an icosahedron (20 faces, 12 vertices and 30 edges) as the base shape and performed 2 levels of subdivision so the final design has 320 faces, 162 vertices and 480 edges. The sensor layout is shown in Figure 6(a). Secondly, the addition of extra analog to digital converters (ADC's) in a confined location technically required very careful design and construction to accommodate the dense electronics.

\subsection{Foam Construction}

Insulating the individual foam sensors and maintaining good contact at the termination points is critical to the performance of each sensor. Previously we created each sensor separately then attached them to the plastic sphere. This approach is tedious and not scalable as the number of sensors is increased. To overcome this problem we have used a technique that uses liquid foam poured into a custom mould (as shown in Figure 6(b)). Smooth On's FlexFoam-iT! III ${ }^{5}$ liquid foam was poured into the custom mould to create the insulating and structural part of the foam sensor as shown in Figure 6(c). Once the molding process is completed, individual conducting foam inserts are placed into each of the holes (Figure 6(d)). We constructed the insulating part of sphere's foam surface in two halves to ease the complexity of construction and allow assembly and dis-assembly of the final input device.

\footnotetext{
${ }^{4}$ ftp://ftp.sgi.com/graphics/SGIIMAGESPEC

${ }^{5}$ http://www.smooth-on.com/
}

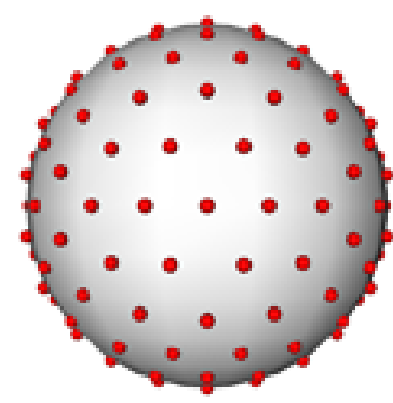

(a)

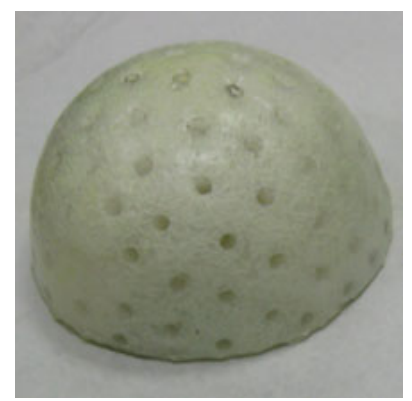

(c)

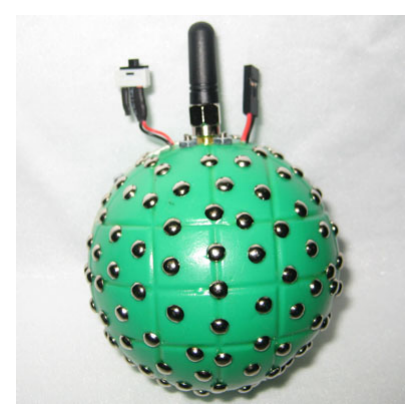

(e)

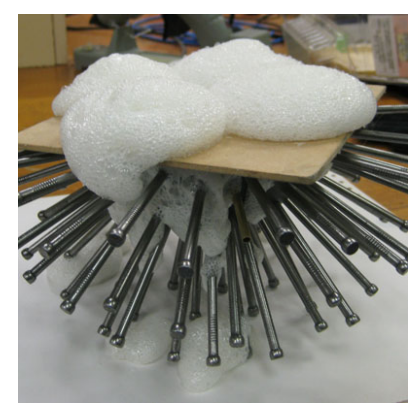

(b)

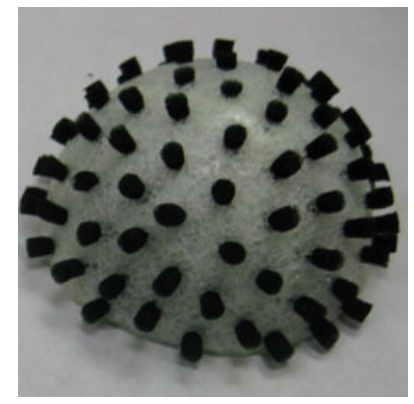

(d)

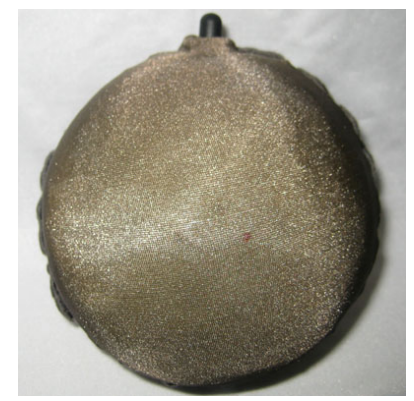

(f)
Figure 6: (a) Layout of 162 evenly spaced sensors on the sphere's surface generated using a subdivision algorithm. (b) Custom mold used for casting foam. (c) Custom cast foam. (d) Conductive foam inserts inserted into foam mold. (e) Complete electronics with exposed terminals, on/off switch, antenna and charging port. (f) Constructed Spherical Digital Foam input device.

\subsection{Electronics}

The electronics used in the new 162 sensor Spherical Digital Foam prop are similar in design to the schematics used on the previously presented 21 sensor Spherical Digital Foam prop [Smith et al. 2008]. Additional ADCs were added to measure the resistance of each conductive foam sensors, to support the increased number of foam sensors. In total there are 16 TLV1543s each with 11 channels that allow the capture of a 10 bit sample for each of the 162 foam sensors (Exposed sensor terminals shown in Figure 6(e)). Separate boards were created for each ADC chip and attached to the inner surface of the plastic skeleton; this was done to optimize space usage within the sphere. Each ADC chip is connected to a common serial data bus that is managed with a MSP430F1232 microcontroller $^{6}$. Wireless communications to the microcontroller are performed using a Parani-ESD2107 bluetooth module. This module

\footnotetext{
${ }^{6}$ http://www.ti.com/

${ }^{7}$ http://www.sena.com/
} 
was chosen in place of the Promi ESD02 because of the external antenna. With 21 sensor spherical prop, a set of small holes were cut in the outer conductive fabric layer to allow bluetooth signals to be transmitted. Although this worked, we found that when holding the input device with two hands many of the holes would become covered and the signal was significantly attenuated causing slower update rates. To overcome this problem, an external antenna now protrudes though the conductive fabric outer (Figure 6(f)). The signal loss is no longer a problem using the new design. The antenna location also doubles as a reference orientation marker as previously discussed. A 600mAh Lithium-ion battery provides approximately 6 hour of use before charging is required. Finally, the Inertia Cube 2 was upgraded to an Inertia $\mathrm{Cube}^{8}$ allowing additional room inside the plastic sphere to accommodate the dense electronics.

\subsection{Dynamic Button Allocation}

The creation of the new higher resolution Spherical Digital Foam has inspired a new technique concept not possible using the original Spherical Digital Foam [Smith et al. 2008]. We propose unique areas of the foam's surface that can be configured in run-time to set up active regions for different operations. For example, the system prompts the user to configure a "left click", in turn the user would depress the desirable area of the spherical prop for their personalized "left click" operation. The application would then record the surface selected and the pressure used so as intelligent decisions could be made to interpret a "left click" operation. Some kind of intelligent reasoning techniques such as Hidden Markov Models might be applied.

\section{User Evaluation}

We conducted a trial study to gather qualitative user experience information when using a Spherical Digital Foam input device to control a menu system. Five participants took part in the study, 4 male and 1 female with ages from 20 to 29 . Each subject was asked to use a Digital Foam input device to navigate through through five separate menu options.

The experiment was conducted in the following order. The input device was described and subjects were given time to familiarize themselves with the Digital Foam menu interface. The menu was configured to have five menu items (red, green, blue, black and white) each which change the color of a large sphere in the center of the screen. Each subject was then asked to perform two tasks. First, participants were invited to hold the Spherical Digital Foam with one hand to select an ordered series of options from the menu. For the second task the menu system maintained the same configuration and participants were asked to hold the Spherical Digital Foam input device with two hands and select another ordered series of menu options. Once completed subjects were asked to fill in a questionnaire with 9 questions (shown in Table 1) with a Likert scale from 1 - 5 where 1 = very easy, too small, strongly agree and $5=$ very hard, too large, strongly disagree.

The results of this study provided valuable feedback for the future Digital Foam design parameters. Due to the shielded design of Digital Foam's conductive fabric material (described [Smith et al. 2008]), the Inertia Cube's magnetometer does not read a strong magnetic north value causing the orientation tracking to drift. This technical problem made menu selection more difficult. When selecting menu items, drifting orientation values occasionally caused the incorrect item to be selected. If this occurred the instant before a click event, an incorrect menu item would be selected. One user suggested not accepting the click event if the menu item had

\footnotetext{
${ }^{8}$ http://www.intersense.com/
}

\begin{tabular}{lc}
\hline Question & Average \\
\hline Q1: Displaying (turning on) the menu was: & 1.0 \\
Q2: Performing a click using Digital Foam: & 3.4 \\
Q3: Selecting the correct menu item was: & 3.8 \\
Q4: I found the input device: & 3.4 \\
Q5: The rotation angle to change menu items was: & 3.6 \\
Q6: I found operating menus with one hand: & 3.8 \\
Q7: I found operating menus with two hands: & 1.8 \\
Q8: Overall I found the menu easy to use: & 3.0 \\
Q9: Overall I could control the menu system: & 2.0 \\
\hline
\end{tabular}

Table 1: Digital Foam Menu User Evaluation Questions

only been highlighted for very short time. Although the drift affected the accuracy of selecting options when asked if the rotation angle to transition between menu options was too small or too large (Table 1 Q5) both the average response (3.6) and user comments indicated a smaller transition angle is desirable. From this we note the importance of the orientation tracking resolution and performance and endeavor to improve tracking performance on future iterations. Another notable feature reliant on orientation tracking is the up-side-down menu entering technique, users operation of this technique appeared unaffected by the orientation drift. All subjects indicated that it was very easy to enter the menu mode by turning the Spherical Digital Foam input device up-side-down (Table 1 Q1). We note the importance of tracker resolution relative to the user control.

The $11 \mathrm{~cm}$ diameter of the Spherical Digital Foam input device is a characteristic we would like to further explore. When asked if the input device was too small or to large (Table $1 \mathrm{Q} 4$ ) the average (3.4) and user comments indicated a smaller size would be more comfortable and easier to use. The comfort of input device is important, Spherical Digital Foam is designed to be hand held but the current size is not optimal. A useful suggestion for future design is to reference the size of commonly used performers juggling balls. Common sizes range from 2" $(5 \mathrm{~cm})$ to 4 " $(10 \mathrm{~cm})$ and could be used as a base line for future development characteristics.

All subjects indicated that operating the menu with one hand was difficult in comparison to using two hands (Table 1 Q6 and Q7). We observed that while using two hands users would shuffle the input device while scrolling through menu options. Where as when using one hand this is a difficult operation. Altering the menus up-sidedown entering operation and reducing the transition angle for menu item transitions may improve the performance for single handed operation although this is not a currently significant requirement.

\section{Conclusion}

Techniques presented in this paper have been developed to support 3D modeling tasks using Spherical Digital Foam as a sole input device. We have extended the "free-form sculpting and clutching" technique to allow surface manipulation of existing 3D models using a mapping between the hardware device and model. Additionally, a tilt operated clutching mechanism was implemented allowing the operations to be cumalitive. We have presented and implemented the half hemisphere technique designed to assist a user while performing sculpting operations. This allows more intuitive operations to be performed by filtering unwanted press locations on the Digital Foam's surface. We also presented the implementation of a camera view control mechanism that allows a user to simultaneously move the camera around in an orbital fashion whilst controlling the zoom parameter with the touch pressure. A menu control system was presented that allows Spherical Digital Foam to perform command and control operations as a sole input device. 
The design and construction of a 162 sensor resolution Spherical Digital Foam input device is also presented. We discuss design consideration and new scalable implementation techniques for the future development of Digital Foam sensors. The new input device has also inspired a new dynamic button allocation technique to facilitate configurable command and control operations to be proposed.

We presented a trial study that has evaluated qualitative aspects of the menu system developed for Digital Foam. The menu system was well received, but a limitation in the sensing of the orientation caused a number of problems with menu item selection. Trial study results will facilitate new prototype, technique and user evaluation design directions. Finally, we intend to investigate the use of Digital Foam and the integration of existing modeling techniques, such as mesh selection. A number of interesting questions have been raised as to how complex non-solid or non-spherical virtual shapes can be mapped to the physical shape of the Spherical Digital Foam input device.

\section{References}

Blaskö, G., AND FeINER, S. 2002. A menu interface for wearable computing. In 6th International Symposium on Wearable Computers (ISWC 2002), 164-165.

Bowman, D. A., And Wingrave, C. A. 2001. Design and evaluation of menu systems for immersive virtual environments. In Proceedings of IEEE Virtual Reality, 149-156.

BRAdY, S., DiAmond, D., AND LAU, K.-T. 2005. Inherently conducting polymer modified polyurethane smart foam for pressure sensing. Sensors and Actuators A: Physical 119, 2 (Apr), 398-404.

BuXton, W. 1995. ”Touch, Gesture, and Marking” Readings in Human Computer Interaction: Toward the Year 2000.

DEERING, M. F. 1995. Holosketch: A virtual reality sketchin / animation tool. ACM Transactions on Computer-Human Interaction 2, 3 (Sep), 220-238.

FröEhlich, B., AND Plate, J. 2000. The cubic mouse a new device for three-dimensional input. In Proceedings of the SIGCHI conference on Human factors in computing systems.

Gerber, D., And Bechmann, D. 2004. Design and evaluation of the ring menu in virtual environments. In Immersive projection technologies.

Gerber, D., And Bechmann, D. 2005. The spin menu: a menu system for virtual environments. In IEEE Virtual Reality Conference, 271-272.

Hinkley, K., AND Sinclair, M. 1999. Touch-sensing input devices. In Proceedings of the SIGCHI conference on Human factors in computing systems, 223-230.

Hinkley, K., Pausch, R., Goble, J. C., And Kassell, N. F. 1994. Passive real-world interface for neurosurgical visualization. In Proceedings of the SIGCHI conference on Human factors in computing systems, 452-458.

Hinkley, K., Pausch, R., Proffitt, D., And Kassell, N. F. 1998. Two-handed virtual manipulation. ACM Transactions on Computer-Human Interaction 5, 3, 260-302.

Jung, H.-K., NAM, T.-J., LeE, H.-S., AND HAN, S.-Y. 2004. Spray modeling: Augmented reality based 3d modeling interface for intuitive and evolutionary form development. In In Proceedings of International Conference on Artifical Reality and Telexistence.

JUNG, H.-K., NAM, T.-J., AND LEE, H.-S. 2005. 3d modeling interface with air spray: Field study of $3 \mathrm{~d}$ model making and prototype development. In Conference On Human Factors In Computing Systems.

KamiYAma, K., KaJimoto, H., VlaCk, K., KaWAKami, N., Mizota, T., AND TACHI, S. 2004. Gelforce. In ACM SIGGRAPH 2004 Emerging techologies, 5.

Koller, D. R., Mine, M. R., And Hudson, S. E. 1996. Headtracked orbital viewing: An interaction technique for immersive virtual environments. In The 28th Intl. Conference on Computer Graphics and Interactive Techniques, 81-82.

Kruger, W., AND FrÖEhlich, B. 1994. The responsive workbench. In IEEE Computer Graphics and Applications, 12-15.

Kulik, A., BlaCh, R., AND FrÖEHLICH, B. 2006. "two - 4 six" - a handheld device for 3D-presentations. In IEEE Symposium on 3D User Interfaces, 167-170.

LiANG, J., AND GREEN, M. 1993. Geometric modeling using siz degrees of fredom input devices. In Proc. 3rd International Conference on CAD and Computer Graphics, 217-222.

MASSIE, T. H., AND SALISBURY, J. K. 1994. The phantom haptic interface: A device for probing virtual objects. In ASME Winter Annual Meeting, Symposium on Haptic Interfaces for Virtual Environment and Teleoperator Systems.

McDonnell, K. T., QIn, H., And WlodarczyK, R. A. 2001. Virtual clay: A real-time sculpting system with haptic toolkits. In Proceedings of the 2001 symposium on Interactive $3 D$ graphics, 179-190.

Milczynski, M., Hermann, T., Bovermann, T., And RitTER, H. 2006. A malleable device with applications to sonification-based data exploration. In Proceedings of the 12th Meeting of the International Conference on Auditory Display.

PIEKARSKI, W., AND SMITH, R. 2006. Robust gloves for $3 \mathrm{~d}$ interaction in mobile outdoor ar environments. In International Symposium on Mixed and Augmented Reality.

PIEKARSKI, W., AND Thomas, B. H. 2004. Augmented reality working planes: A foundation for action and construction at a distance. In Proceedings of the Third IEEE and ACM International Symposium on Mixed and Augmented Reality, 162-171.

Reitmayr, G., Chiu, C., Kusternig, A., Kusternig, M., AND WitZMANN, H. 2005. iorb - unifying command and 3d input for mobile augmented reality. In $3 D$ User Interfaces.

Schkolne, S., Pruett, M., AND Schröder, P. 2001. Surface drawing: creating organic $3 \mathrm{~d}$ shapes with the hand and tangible tools. In Proceedings of the SIGCHI conference on Human factors in computing systems, 261-268.

Smith, R. T., Thomas, B. H., AND Piekarski, W. 2008. Tech note: Digital foam. In IEEE Symposium on 3D User Interfaces.

Vogt, F., Chen, T., Hoskinson, R., And Fels, S. 2004. A malleable surface touch interface. In Technical Sketch. Sketches and Applications at ACM SIGGRAPH.

Woo, M., NeIder, J., AND Davis, H. 1998. OpenGL Programming Guide, Second Edition, The Official Guide to learning Open GL, Version 1.1. 